patients with systemic lupus erythematosus (SLE). A team of Canadian researchers, therefore, investigated whether a high serum total cholesterol level in SLE patients is associated with poor outcomes-specifically, renal deterioration, death from renal involvement, and end-stage renal disease (ESRD).

The researchers analyzed first available serum samples and data from 1,060 patients (88\% female) at a single Canadian SLE clinic. Overall, outcome-free survival estimates for each of the three outcomes were markedly worse for patients with a total cholesterol level of $>5.2 \mathrm{nmol} / \mathrm{l}$. Univariate analysis showed that for every $1 \mathrm{mmol} / \mathrm{l}$ increase in total cholesterol level, there was a $27 \%$ increase in the hazard ratio (HR) of renal deterioration $(P<0.0001)$. On multivariate analysis, total cholesterol level was associated with renal deterioration (HR 1.17, 95\% Cl 1.01-1.36, $P=0.0374$ ) and death from renal involvement (HR 1.33, 95\% Cl 1.20-1.47, $P<0.0001)$, but not ESRD. As ESRD was present in only $4 \%$ of the sample, this failure to reach significance might simply be the result of insufficient power.

These findings suggest that cholesterol contributes to renal damage, and the researchers recommend that the role of dyslipidemia in the pathogenesis of renal disease be further investigated.

Original article Tisseverasinghe A et al. (2006) Association between serum total cholesterol level and renal outcome in systemic lupus erythematosus. Arthritis Rheum 54: 2211-2219

\section{Increased mortality rate in patients with RA after a first cardiovascular event}

It is well known that patients with rheumatoid arthritis (RA) have a higher incidence of cardiovascular events than people without RA; however, the risk of death following a cardiovascular event in patients with RA, compared with the general population, has not previously been investigated. Van Doornum and colleagues have now shown that after a first cardiovascular event, patients with RA have a higher mortality rate than people without RA.

The data for this retrospective cohort study were obtained between July 2001 and December 2003 from The Victorian Linked Dataset, which includes information about all episodes of hospital patient care and deaths in Victoria, Australia. According to this dataset, 29,924 patients experienced a first cardiovascular event (myocardial infarction or stroke), of whom 359 had a diagnosis of RA. The 30-day cardiovascular mortality rate was $17.6 \%$ for patients with RA, compared with $10.9 \%$ for patients without $\mathrm{RA}$; the excess mortality in RA patients was almost entirely attributable to an increased number of deaths from myocardial infarction, rather than from stroke. The higher fatality rate in RA patients did not change after adjustment for traditional cardiovascular risk factors, comorbidity and socioeconomic status.

The authors speculate that the increased cardiovascular mortality rate in patients with RA could be caused by the treatments for $R A$, systemic inflammation, delay in seeking medical attention and in diagnosis, and differences in treatments administered after cardiovascular events; however, the data obtained could not directly address these issues, and further research is required to identify the mechanisms responsible.

Original article Van Doornum S et al. (2006) Increased case fatality rates following a first acute cardiovascular event in patients with rheumatoid arthritis. Arthritis Rheum 54: 2061-2068

\section{Genetic profile predicts response to infliximab treatment for RA}

Between $20 \%$ and $40 \%$ of patients with rheumatoid arthritis (RA) who receive therapy with methotrexate and a tumor necrosis factor inhibitor (such as infliximab) do not respond to treatment. As the prognostic markers usually used in RA cannot predict patient responses to this therapy, some researchers have turned to genetic profiling. Lequerré and colleagues have now identified two sets of transcripts that can reliably predict patient response to methotrexate-infliximab treatment.

Transcriptome analysis of the peripheral blood mononuclear cells of 33 patients with methotrexate-refractory RA was performed at baseline. Patients then underwent treatment with infliximab, and were considered to have responded to this therapy if they showed an improvement of 1.2 in the Disease Activity Score 28 after 3 months. Lequerré and colleagues identified a final set of 20 transcripts that predicted treatment response with $90 \%$ 